\title{
Bias-Corrected Estimation in Continuous Sampling Plans
}

\author{
Geoffrey Decrouez, ${ }^{1}$ Andrew Robinson ${ }^{2}$
}

${ }^{1}$ National Research University, Higher School of Economics, Moscow, Russia, ggdecrouez@hse.ru

${ }^{2}$ CEBRA \& School of Mathematics and Statistics, University of Melbourne, Australia, apro@unimelb.edu.au

Continuous sampling plans (CSP) are algorithms used for monitoring and maintaining the quality of a production line. Although considerable work has been done on the development of CSP plans, to our knowledge there has been no corresponding effort in developing estimators with good statistical properties for data arising from a CSP inspection process. For example, information about the failure rate of the process will affect the management of the process, both in terms of selecting appropriate CSP parameters to keep the failure rate after inspection at a suitable level, and in terms of policy, for example whether the process should be completely inspected, or shut down. The motivation for this exercise was developing sampling protocols for Australia's Department of Agriculture and Water Resources for monitoring the biosecurity compliance of incoming goods at international borders. In this study we show that maximum likelihood estimation of the failure rate under a CSP sampling scheme can be biased depending on when estimation is performed, and we provide explicit expressions for the main contribution of the bias under various CSP sampling plans. We then construct biascorrected estimators and confidence intervals, and evaluate their performance in a numerical study.

KEY WORDS: point and interval estimation, border inspection, continuous sampling plans

This is the author manuscript accepted for publication and has undergone full peer review but has not been through the copyediting, typesetting, pagination and proofreading process, which may lead to differences between this version and the Version of Record. Please cite this article as doi: $10.1111 /$ risa.12811

This article is protected by copyright. All rights reserved. 


\section{Introduction}

The Australian Federal Department of Agriculture and Water Resources (the department) carries out border biosecurity inspections of incoming cargo, mail, ocean vessels, and international passengers, both to prevent entry of regulated pests and to verify the compliance of these pathways to biosecurity regulations. The department uses Continuous Sampling Protocols (CSP, first introduced in ${ }^{(1,2)}$ and described in the next section) on pathways that it deems to represent a low risk of biosecurity contamination. The department needs to evaluate the biosecurity risk presented by each pathway, and uses the contamination rate, or the rate at which units on the pathway are compromised, as one metric. When a pathway is either fully inspected or sampled randomly, the evaluation of the non-compliance rate is comparatively straightforward. However, for the low-risk pathways for which the department uses CSP, estimating the non-compliance rate is more complex. This paper describes a project undertaken to provide the department with high-quality estimators of the non-compliance rate for the apparently low-risk pathways that are managed using a CSP; see for example http://www.daff.gov.au/biosecurity/import/plant-products/risk-return. These are needed by biosecurity regulators to prioritize pathways for inspection; to alert responsible authorities about changes in pathway performance; and to assess the suitability of upstream risk mitigation procedures such as offshore audits and third-party on-site inspections.

Although this paper is motivated by border inspection for biosecurity as is performed by the department and other biosecurity regulators to detect and exclude biosecurity contamination, CSP methods are broadly applied, for example in the US military ${ }^{(3)}$, originally to monitor the proportion of defects present in military armaments manufactured by government contractors; for auditing in double data entry ${ }^{(4)}$ or outcomes of online transactions ${ }^{(5)}$; fraud detection $^{(6)}$; and construction, e.g. of circuit boards ${ }^{(7)}$.

We define inspection as the examination of an individual unit to see whether or not it is compliant with relevant regulations. Typically an inspection will determine whether the unit contains biosecurity risk material or contamination. More generally, a unit could be an international passenger, a sea container, or a pallet of goods. To keep our exposition as general as possible, we will refer to a unit as non-compliant under the following circumstances: (i) biosecurity contamination is detected, (ii) the unit is mislabelled or (iii) the label is missing. Throughout this study, we assume perfect in- 
spection, that is, non-compliance in a unit that is inspected will certainly be detected. This assumption is unlikely to be true in operational settings but we believe that our conclusions are robust to the assumption.

We model successive units arriving at the border as a time series. Let $p_{i}$ be the probability that the $i$-th unit is non-compliant (the non-compliance rate). Different time-series models for this kind of border inspection data, including a Bernoulli process, a Markov chain, and a hidden Markov model, were considered in ${ }^{(8)}$. The authors showed that the Bernoulli process is a reasonable model to describe the dynamics of the evolution of the status of incoming units with time, when the models are fit to inspection data. The Bernoulli model assumes mutual independence between successive units, and assumes that the non-compliance rate is constant, with $p_{i}=p$ for all $i$.

In this study we address the problem of estimating $p$ under the Bernoulli model when a continuous sampling plan is implemented, and estimation is performed at the end of a CSP cycle. This might occur in a review of a pathway's performance to assess the appropriateness of the design of the intervention. We show that the maximum likelihood estimator is biased, suggest a bias correction, and study the performance of the bias-corrected estimator. The robustness of the bias correction under a time-varying noncompliance rate and correlated observations is also assessed in a numerical study.

We were able to find little previous work on the issue of bias correction in CSP ${ }^{(1,2)}$. Previous studies focus on estimation of the average outgoing quality (AOQ), which integrates the non-compliance rate, the sampling program, and inspection quality. However, the contamination rate must be known or estimated in order to use the AOQ. Maximum likelihood estimation of the non-compliance rate at the end of a census cycle, under a Markov chain model for the observation process, is briefly mentioned in ${ }^{(9)}$. However, they did not study the properties of the estimator. For recent contributions on continuous sampling plans, see $(10,11,12,13,14)$.

The paper is organised as follows. In $₫ 2$ we describe the sampling plan procedures, and we present a novel way to represent them using random vectors. The distribution of the random vectors is derived in \$3. Estimators and bias-corrected estimators are introduced in $\$ 4$, and their performance and robustness is evaluated in a numerical study. The problem of construction of confidence intervals is addressed in \$5. Finally, an application to border biosecurity inspections is discussed in \$6, 


\section{Sampling plan procedures}

In this section we present a scheme that was introduced in (1) under the name CSP-1 for monitoring the quality of a production line, and two of its generalisations, known as CSP-2 and CSP-3, introduced in ${ }^{(2)}$. We also establish notation that will be used in the subsequent sections.

\subsection{Census mode}

Census mode is common to all sampling plans. During census, $100 \%$ of the items are inspected, as they arrive at the border. Census mode terminates when $r$ consecutive units pass inspection. The number $r$ is referred to as the clearance number. Let $Z_{i}$ be the status of unit $i$, set to 0 if compliant and 1 if non-compliant and let $N_{0}$ be the total number of units inspected in census mode before $r$ successive units are found clear of defects. A failure sequence is a sequence of at most $r-1$ consecutive zeros, followed by a non-compliant unit. We can express $N_{0}=\sum_{j=1}^{r} j X_{j}+r$, where $X_{j}$ is the number of failure sequences of length $j$ before the first occurrence of $r$ consecutive items clear of defects. For example, if the inspectorate observes

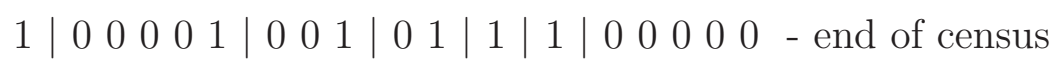

with $r=5$, then there are 6 failure sequences, and $X_{1}=3, X_{2}=X_{3}=$ $X_{5}=1$, and $X_{4}=0$. A census cycle is fully described by the random vector $\mathbf{X}=\left(X_{1}, \ldots, X_{r}\right)$.

\subsection{Continuous Sampling Plan 1}

CSP-1 is defined by two inspection modes (census and sampling), with switching rules to decide when to shift between them:

1. Census mode: While the number of consecutive items to pass inspection is less than the clearance number $r$, inspect all items. When $r$ consecutive items have passed inspection, go to step 2.

2. Sampling mode: While all inspected items pass inspection, inspect a fraction $f$ of the units. When an item fails inspection, return to step 1 .

A CSP-1 cycle starts in census mode. Note that the two control parameters $r$ and $f$ are defined by the user, and can be chosen on the basis of several different performance measures, such as the $\mathrm{AOQ}^{(1)}$. The number of items 
inspected during sampling mode is denoted by the random variable $S$. The sampling mode $s$ in a CSP-1 cycle typically looks like

$$
s: 00000001 \text { - return to census, }
$$

for which $S=8$ here. The number of inspected items in one CSP-1 cycle is $N_{1}:=N_{0}+S$. A CSP-1 cycle is thus fully described by the random vector $\left(X_{1}, \ldots, X_{r}, S\right)$. It will be convenient later when discussing CSP-1, CSP-2 and CSP-3 to use notation $\mathbf{X}=\left(X_{1}, \ldots, X_{r}\right)$ to describe the census mode, and notation $\mathbf{L}_{i}$ to describe the remaining modes corresponding to the CSP- $i$ scheme, where $i=1,2,3$. For CSP-1, we have $\mathbf{L}_{1}=S$, so that a CSP-1 cycle is fully described by the random vector $\left(\mathbf{X}, \mathbf{L}_{1}\right)$.

\subsection{Continuous Sampling Plan 2}

An early criticism of CSP-1 was that it is too sensitive to the occasional noncompliant item, hence shifting to census mode more often than necessary. Two plans that reduce the frequency of switches from sampling mode to census mode when unnecessary are proposed in ${ }^{(2)}$. The first of these two plans, CSP-2, runs as follows:

1. Census mode: While the number of consecutive items to pass inspection is less than the clearance number $r$, inspect all items. When $r$ consecutive items have passed inspection, go to step 2.

2. Sampling mode: While all inspected items pass inspection, inspect a fraction $f$ of the units. When an item fails inspection, go to step 3 .

3. Alert mode: While the number of items to pass inspection is less than $k$, and no items fail inspection, inspect a fraction $f$ of the units. If an item fails inspection, return to step 1 . Otherwise, when $k$ items have passed inspection, return to step 2 .

Let $N_{a}$ be the number of times we go through the sampling and alert modes, let $S_{i}$ be the number of items inspected during the $i$-th sampling mode, and let $W_{a}$ be the number of items inspected in the last alert mode, before returning to census mode. The total number of units inspected during sampling mode is $S^{N_{a}}:=\sum_{i=1}^{N_{a}} S_{i}$. A CSP-2 cycle is fully described by the random vector $\left(\mathbf{X}, \mathbf{L}_{2}\right)$, where $\mathbf{L}_{2}:=\left(S_{1}, \ldots, S_{N_{a}}, N_{a}, W_{a}\right)$. An example of a sequence of observations during sampling and alert modes ( $s$ and $a$ modes) with $k=3$ 
is given below,

$$
s: 000001|a: 0000| s: 0001 \mid a: 01 \text {, }
$$

for which $S_{1}=5, S_{2}=4, N_{a}=2$, and $W_{a}=2$. The total number of units inspected in one CSP-2 cycle is $N_{2}:=N_{0}+S^{N_{a}}+\left(N_{a}-1\right) k+W_{a}$.

\subsection{Continuous Sampling Plan 3}

The second plan is called CSP-3 ${ }^{(2)}$. It follows the same basic process as CSP2 , except that immediately after a switch to alert mode, the next $l$ items to arrive are all inspected, before the usual alert mode sampling begins. The algorithm for CSP-3 can be expressed as follows:

1. Census mode: While the number of consecutive items to pass inspection is less than the clearance number $r$, inspect all items. When $r$ consecutive items have passed inspection, go to step 2.

2. Sampling mode: While all inspected items pass inspection, inspect a fraction $f$ of the units. When an item fails inspection, go to step 3 .

3. Limbo mode: Inspect the next $l$ items. If an item fails inspection, return to step 1. Otherwise, if all $l$ items pass inspection, go to step 4 .

4. Alert mode: While the number of items to pass inspection is less than $k$, and no items fails inspection, inspect a fraction $f$ of the units. If an item fails inspection, return to step 1. Otherwise, when $k$ items have passed inspection, return to step 2.

Let $N_{l}$ and $N_{a}$ be the number of times we go through the limbo and alert mode. Note that since a sampling mode is necessarily followed by a limbo mode, the number of times we go through the sampling mode is $N_{l}$. Let $S_{i}$ be the number of items inspected during the $i$-th sampling mode, for $i=1, \ldots, N_{l}$. Finally, let $W_{a}$ be the number of items inspected in the last alert mode, before returning to census mode $\left(W_{a}=0\right.$ if we return to census from limbo mode), and let $W_{l}$ be the number of non-compliant units in the last limbo mode, before returning to census $\left(W_{l}=0\right.$ if we return to census from alert mode). A CSP-3 cycle is fully described by the random vector $\left(\mathbf{X}, \mathbf{L}_{3}\right)$, where $\mathbf{L}_{3}:=\left(S_{1}, \ldots, S_{N_{l}}, N_{l}, N_{a}, W_{l}, W_{a}\right)$. Return to census mode can occur either from limbo or alert mode ( $l$ and $a$ modes). We illustrate this below, assuming the inspectorate collects the following data with $l=4$ 
and $k=3$,

$$
\begin{array}{rl}
s: 000 & 1|l: 0000| a: 000 \mid s: 001
\end{array}
$$

In both cases we go through two limbo modes, so that $N_{l}=2, S_{1}=4$ and $S_{2}=3$. In scenario (i), inspection of the next 4 items reveals 2 non compliant units, so that the inspectorate switches back to census mode from limbo mode, and $W_{l}=2, W_{a}=0$ and $N_{a}=1$. In the second scenario, the switch occurs during alert mode, and $W_{l}=0, W_{a}=2$ and $N_{a}=2$. The number

of inspections during sampling mode is thus $S^{N_{l}}:=\sum_{i=1}^{N_{l}} S_{i}$, and the total number of inspections in one CSP-3 cycle is $N_{3}:=N_{0}+S^{N_{l}}+(k+l) N_{l}+W_{a}-k$.

\section{Distribution of $\left(\mathrm{X}, \mathbf{L}_{i}\right)$}

We derive the distribution of the random vectors $\left(\mathbf{X}, \mathbf{L}_{i}\right)$ under the assumption that observations $Z_{i}$ are i.i.d. Bernoulli random variables, with $\mathbf{P}\left(Z_{i}=1\right)=p=1-\mathbf{P}\left(Z_{i}=0\right)$. Under this assumption, $\mathbf{X}$ and $\mathbf{L}_{i}$ are independent. The distributions given here will be used in the next section to derive the maximum likelihood estimator of $p$ and some of its properties.

\subsection{Distribution of $\mathrm{X}$}

The event consisting of the arrival of $r$ consecutive clear items occurs with probability $q^{r}$, where $q:=1-p$. Recall that $X_{j}$ is the number of failure sequences of length $j$, for $1 \leq j \leq r$, before the first appearance of $r$ consecutive clear items. The probability of a failure sequence of length $j$ is $p_{j}:=p q^{j-1}$. We are in the setting of waiting times in multiple Bernoulli trials. The probability of a sequence containing $X_{1}=x_{1}$ failures sequences of length $1, X_{2}=x_{2}$ failures sequences of length 2 , all the way to $X_{r}=x_{r}$ failures sequences of length $r$, followed by $r$ consecutive clear items is $p_{1}^{x_{1}} \times \ldots \times p_{r}^{x_{r}} \times q^{r}$. Since 
there are

$$
\begin{aligned}
\left(\begin{array}{c}
x_{1}+\ldots+x_{r} \\
x_{1}
\end{array}\right)\left(\begin{array}{c}
x_{2}+\ldots+x_{r} \\
x_{2}
\end{array}\right) \\
\quad \times \ldots \times\left(\begin{array}{c}
x_{r-1}+x_{r} \\
x_{r-1}
\end{array}\right)=\frac{\left(\sum_{j=1}^{r} x_{j}\right) !}{x_{1} ! \times \ldots \times x_{r} !}
\end{aligned}
$$

ways of rearranging the sequence, the joint distribution of $\mathbf{X}$ is

$$
\mathbf{P}(\mathbf{X}=\mathbf{x})=\frac{\left(\sum_{j=1}^{r} x_{j}\right) !}{\prod_{j=1}^{r} x_{j} !} q^{r}\left(\prod_{j=1}^{r} p_{j}^{x_{j}}\right)
$$

where $\mathbf{x}=\left(x_{1}, \ldots, x_{r}\right)$, which corresponds to a negative multinomial distribution with parameters 1 and $\mathbf{p}:=\left(p_{1}, \ldots, p_{r}\right)$, where $\sum_{j=1}^{r} p_{j}=1-q^{r}$. The marginal distribution of $X_{j}$ is negative binomial, with $\mathbf{P}\left(X_{j}=x_{j}\right)=$ $\left(\begin{array}{c}1+x_{j}-1 \\ x_{j}\end{array}\right) s_{j}\left(1-s_{j}\right)^{x_{j}}$. In particular, $\mathbf{E}\left(X_{j}\right)=p_{j} / q^{r}$, where $\mathbf{E}(\cdot)$ denotes the expected value.

\subsection{Distribution of $\mathrm{L}_{1}$}

Under the Bernoulli assumption, $\mathbf{L}_{1}=S$ represents the waiting time until the first detection of a non-compliant item, and is geometrically distributed, with $\mathbf{P}(S=s)=p q^{s-1}$, for $s \geq 1$, and mean $\mathbf{E}(S)=1 / p$.

\subsection{Distribution of $\mathrm{L}_{2}$}

Under the CSP-2 sampling plan, the random variables $S_{i}$ are geometrically distributed, with $\mathbf{P}\left(S_{1}=s_{1}\right)=p q^{s_{1}-1}$, for $s_{1} \geq 1$ and $\mathbf{E}\left(S_{1}\right)=1 / p$. The probability that we return to sampling mode from alert mode is $q^{k}$, which is the probability that the $k$ items inspected during alert mode are clean. The number $N_{a}$ of sampling cycles thus has a geometric distribution, with $\mathbf{P}\left(N_{a}=n\right)=\left(1-q^{k}\right) q^{k(n-1)}$, for $n \geq 1$ and $\mathbf{E}\left(N_{a}\right)=1 /\left(1-q^{k}\right)$. The mean of $S^{N_{a}}$, the total number of units inspected during sampling mode, is $\mathbf{E}\left(S^{N_{a}}\right)=$ $\mathbf{E}\left(N_{a}\right) \mathbf{E}\left(S_{1}\right)=1 /\left(p\left(1-q^{k}\right)\right)$. Finally, the number of items inspected during the last alert mode is by definition less than $k$, its distribution is thus given by $\mathbf{P}\left(W_{a}=w\right)=\mathbf{P}(Y=w \mid Y \leq k)$, where $Y$ follows a geometric distribution 
with parameter $p$. We get that

$$
\mathbf{P}\left(W_{a}=w\right)=\frac{\mathbf{P}(Y=w)}{\mathbf{P}(Y \leq k)}=\frac{p q^{w-1}}{1-q^{k}},
$$

for $1 \leq w \leq k$, with mean

$$
\mathbf{E}\left(W_{a}\right)=\frac{p}{1-q^{k}} \sum_{w=1}^{k} w q^{w-1}=\frac{1}{p}-\frac{k q^{k}}{1-q^{k}}
$$

\subsection{Distribution of $\mathrm{L}_{3}$}

The distribution of the number of items inspected in one sampling mode is the same as for CSP-1 and 2, specifically, $\mathbf{P}\left(S_{1}=s_{1}\right)=p q^{s_{1}-1}$, for $s_{1} \geq 1$, $\mathbf{E}\left(S_{1}\right)=1 / p$. The derivation of the distribution of $\mathbf{L}_{3}$ is more complicated here because the design of CSP-3 introduces dependence between the random variables $N_{l}, N_{a}, W_{l}$ and $W_{a}$ : the number of times the inspectorate goes through the alert mode is not independent of the number of times it goes through limbo mode. Likewise, $W_{a}$ and $W_{l}$ cannot be non zero at the same time. We refer the reader to $\S 1$ of the Appendix for details.

\section{Point estimation}

We consider maximum likelihood estimation at the end of a CSP cycle: the regulator wishes to estimate the non-compliance rate at the end of the $m$-th CSP cycle corresponding to CSP-1, 2 or 3 . The subscript 0 will refer to estimation at the end of a census mode, and the subscript 1, 2, and 3 to estimation at the end of a full CSP- $i$ cycle, for $i=1,2,3$.

\subsection{Point estimators}

Consider first estimation at the end of a census mode. Let $\mathbf{X}_{1}, \ldots, \mathbf{X}_{m}$ be a random sample of size $m$, where each $\mathbf{X}_{i}=\left(X_{i, 1}, \ldots, X_{i, r}\right)$ is a vector of length $r$, distributed according to (11). The variable $X_{(j)}=\sum_{i=1}^{m} X_{i, j}$ denotes the total number of failure sequences of length $j$ observed over $m$ census cycles. The log-likelihood is $\ell_{0}(p)=\sum_{i=1}^{m} \log \mathbf{P}\left(\mathbf{X}_{i}=\mathbf{x}_{i}\right)$, where $\mathbf{x}_{i}=\left(x_{i, 1}, \ldots, x_{i, r}\right)$, so that ignoring constants independent of the model 
parameters, we obtain

$$
\ell_{0}(p)=\sum_{i=1}^{m} \log \left(\frac{\left(\sum_{j=1}^{r} x_{i, j}\right) !}{\prod_{j=1}^{r} x_{i, j} !} q^{r} \prod_{j=1}^{r} p_{j}^{x_{i, j}}\right)
$$

so that

$$
\begin{aligned}
\ell_{0}(p) & =m r \log (1-p) \\
& +\sum_{j=1}^{r}\left(\sum_{i=1}^{m} x_{i, j}\right)(\log p+(j-1) \log (1-p)) .
\end{aligned}
$$

The maximum likelihood estimator obtained at the end of the $m$-th census cycle is

$$
\hat{p}_{0}=\frac{\sum_{j=1}^{r} X_{(j)}}{m r+\sum_{j=1}^{r} j X_{(j)}},
$$

which corresponds to the number of non-compliant items divided by the total number of inspected consignments.

Similarly, denote by $\ell_{i}(p)$ the log-likelihood of a random sample of size $m$ corresponding to $\mathrm{CSP}-i$, where each component is distributed like a generic $\left(\mathbf{X}, \mathbf{L}_{i}\right)$. For example, the $m$ observations of a generic $N_{a}$ for CSP-2 are denoted $N_{a, 1}, \ldots, N_{a, m}$. Then, $\ell_{i}(p)=\ell_{0}(p)+\xi_{i} \log p+\zeta_{i} \log (1-p)$, with $\xi_{1}=m, \xi_{2}=\sum_{i} N_{a, i}+m, \xi_{3}=\sum_{i} N_{a, i}+\sum_{i} W_{l, i}+m$, and $\zeta_{1}=\sum_{i} S_{i}-m$, $\zeta_{2}=\sum_{i} S_{i}^{N_{a, i}}+(k-1) \sum_{i} N_{a, i}+\sum_{i} W_{a, i}-m(k+1), \zeta_{3}=\sum_{i} S_{i}^{N_{l, i}}+(k+$ l) $\sum_{i} N_{l, i}+\sum_{i} W_{a, i}-m(k+1)-\sum_{i} N_{a, i}-\sum_{i} W_{l, i}$. The maximum likelihood estimator of the non-compliance rate at the end of the $m$-th CSP- $i$ cycle is given by

$$
\hat{p}_{i}=\frac{\sum_{j=1}^{r} X_{(j)}+\xi_{i}}{m r+\sum_{j=1}^{r} j X_{(j)}+\zeta_{i}+\xi_{i}},
$$

which corresponds here as well to the ratio of non-compliant items to the total number of inspected items.

\subsection{Bias corrected estimators}

In the context of continuous sampling plans, the stopping rule is not independent of the observations, and therefore we expect the estimators $\hat{p}_{i}$, 
$i=0, \ldots, 3$ to be biased. Under usual regularity conditions of the loglikelihood ${ }^{(15)}$, the $O\left(\mathrm{~m}^{-1}\right)$ term in the expression of the bias of maximum likelihood estimators can be derived explicitly. In our context, we get

$$
\mathbf{E}\left(\hat{p}_{i}-p\right)=I_{i}^{-2}\left(K_{i} / 2+J_{i}\right)+O\left(m^{-2}\right),
$$

with

$$
\begin{aligned}
& I_{i}=-\mathbf{E}\left(\frac{\partial^{2} \ell_{i}(p)}{\partial p^{2}}\right), \quad K_{i}=\mathbf{E}\left(\frac{\partial^{3} \ell_{i}(p)}{\partial p^{3}}\right), \\
& J_{i}=\mathbf{E}\left(\frac{\partial^{2} \ell_{i}(p)}{\partial p^{2}} \frac{\partial \ell_{i}(p)}{\partial p}\right),
\end{aligned}
$$

where the term $I_{i}^{-2}\left(K_{i} / 2+J_{i}\right)$ is of order $m^{-1}$. The $O\left(m^{-1}\right)$ term can be reexpressed $^{(16)}$ as $\mathbf{E}\left(\hat{p}_{i}-p\right)=I_{i}^{-2}\left(\mathcal{J}_{i}-K_{i} / 2\right)+O\left(m^{-2}\right)=: \mathcal{B}_{i}(p) m^{-1}+O\left(m^{-2}\right)$, where $\mathcal{J}_{i}=-\partial I_{i} / \partial p$. Notation $\mathcal{B}_{i}(p)$ makes explicit the dependence of the bias on $p$, but the $\mathcal{B}_{i}$ terms also depend on other model parameters as well, such as $r, k$ or $l$. To compute the $\mathcal{B}_{i}(p)$ coefficients, we are required to compute the second and third order derivatives of the $\log$-likelihood $\ell_{i}$, which are given by

$$
\begin{aligned}
& \frac{\partial^{2} \ell_{0}(p)}{\partial^{2} p}=-\frac{m r}{(1-p)^{2}}-\sum_{j=1}^{r} X_{(j)}\left(\frac{1}{p^{2}}+\frac{j-1}{(1-p)^{2}}\right), \\
& \frac{\partial^{3} \ell_{0}(p)}{\partial^{3} p}=-\frac{2 m r}{(1-p)^{3}}+2 \sum_{j=1}^{r} X_{(j)}\left(\frac{1}{p^{3}}-\frac{i-1}{(1-p)^{3}}\right),
\end{aligned}
$$

and

$$
\begin{aligned}
& \frac{\partial^{2} \ell_{i}(p)}{\partial^{2} p}=\frac{\partial^{2} \ell_{0}(p)}{\partial^{2} p}-\frac{\xi_{i}}{p^{2}}-\frac{\zeta_{i}}{(1-p)^{2}}, \\
& \frac{\partial^{3} \ell_{i}(p)}{\partial^{3} p}=\frac{\partial^{3} \ell_{0}(p)}{\partial^{3} p}+\frac{2 \xi_{i}}{p^{3}}-\frac{2 \zeta_{i}}{(1-p)^{3}},
\end{aligned}
$$

for $i=1,2,3$. Making use of the expected values derived in $\S 3$ and $\S 1$ of the Appendix, we deduce the expected values of the variables $X_{(j)}, \xi_{i}$ and $\zeta_{i}$, which are given by $\mathbf{E}\left(X_{(j)}\right)=m p q^{j-r-1}$,

$$
\begin{aligned}
& \mathbf{E}\left(\xi_{1}\right)=m, \quad \mathbf{E}\left(\xi_{2}\right)=m\left(\frac{2-q^{k}}{1-q^{k}}\right), \\
& \mathbf{E}\left(\xi_{3}\right)=m\left(1+\frac{q^{l}}{1-q^{k+l}}+\frac{l p}{1-q^{k+l}}\right),
\end{aligned}
$$

This article is protected by copyright. All rights reserved. 
and

$$
\begin{aligned}
& \mathbf{E}\left(\zeta_{1}\right)=\frac{m q}{p}, \quad \mathbf{E}\left(\zeta_{2}\right)=\frac{m q}{p}\left(\frac{2-q^{k}}{1-q^{k}}\right), \\
& \mathbf{E}\left(\zeta_{3}\right)=\frac{m q}{p}\left(\frac{1+q^{l}-q^{k+l}+l p}{1-q^{k+l}}\right) .
\end{aligned}
$$

Together with the expression of the derivatives of the log-likelihood given above and the formula at (5), we obtain after simplification an expression for the terms $I_{i}$ and $K_{i}$,

$$
\begin{aligned}
& I_{0}=\frac{m\left(1-q^{r}\right)}{p^{2} q^{r+1}}, \quad I_{1}=\frac{m}{p^{2} q^{r+1}}, \\
& I_{2}=\frac{\left(1-q^{k}+q^{r}\right) m}{\left(1-q^{k}\right) p^{2} q^{r+1}} \\
& I_{3}=\frac{\left(1-q^{k+l}+q^{r}\left(q^{l}+l p\right)\right) m}{p^{2} q^{r+1}\left(1-q^{k+l}\right)}
\end{aligned}
$$

and

$$
\begin{aligned}
& K_{0}=-\frac{2 m r}{q^{3}}+\frac{2 m\left(1-q^{r}\right)}{p^{3} q^{r}}-\frac{2 m}{p q^{r+3}}\left(q+(r-1) q^{r+1}-r q^{r}\right), \\
& K_{1}=K_{0}+\frac{2(1-2 p) m}{p^{3} q^{2}} \\
& K_{2}=K_{0}+\frac{2(1-2 p)\left(2-q^{k}\right) m}{p^{3} q^{2}\left(1-q^{k}\right)} \\
& K_{3}=K_{0}+\frac{2(1-2 p)\left(1+q^{l}-q^{k+l}+l p\right) m}{p^{3} q^{2}\left(1-q^{k+l}\right)} .
\end{aligned}
$$

Note that $I_{i}^{-1}$ gives the expression of the asymptotic variance of the estimator $\hat{p}_{i}$, which is of order $m^{-1}$. Differentiating the $I_{i}$ terms with respect to $p$ give 
$\mathcal{J}_{i}$, and putting these terms together, we finally obtain

$$
\begin{aligned}
& \mathcal{B}_{0}(p)= \frac{p q^{r}}{1-q^{r}}\left(q-p r \frac{1}{1-q^{r}}\right), \\
& \mathcal{B}_{1}(p)= p q^{r}(q-p r), \\
& \mathcal{B}_{2}(p)= \frac{p q^{r}}{1-q^{k}+q^{r}}\left(q-p r \frac{1-q^{k}}{1-q^{k}+q^{r}}\right)\left(1-q^{k}\right) \\
& \quad+\frac{p q^{r}}{1-q^{k}+q^{r}} k p q^{r+k}, \\
& \mathcal{B}_{3}(p)= \frac{p^{2} q^{2 r}\left(k q^{k+l}+l\left(1+p q^{k}(k+1)\right)\right)}{\left(1-q^{k+l}+q^{r+l}+l p q^{r}\right)^{2}} \\
&+\frac{p q^{r}\left(1-q^{k+l}\right)}{1-q^{k+l}+q^{r+l}+l p q^{r}} \\
& \times\left(q-\frac{p r\left(1-q^{k+l}\right)}{1-q^{k+l}+q^{r+l}+l p q^{r}}\right) .
\end{aligned}
$$

Setting $l=0$ in $\mathcal{B}_{3}(p)$ gives $\mathcal{B}_{2}(p)$, which suggests that CSP-2 can be seen as a special case of CSP-3. Indeed, setting $l=0$ amounts to skipping limbo mode in CSP-3, which is exactly the CSP-2 procedure. However, setting $k=0$ in $\mathcal{B}_{2}(p)$ does not give $\mathcal{B}_{1}(p)$ : CSP-1 is not a special case of CSP-2. By setting $k$ to 0 in CSP-2, we will never go back to census mode since it is not possible to observe a non-compliant item if we do not inspect any: we will return to sampling mode infinitely many times, so that the number of observations is infinite.

Bias-corrected estimators can be constructed using the bias expressions, namely $\mathcal{B}_{0}(p), \ldots, \mathcal{B}_{3}(p)$, and we denote them $\tilde{p}_{i}=\hat{p}_{i}-\mathcal{B}_{i}\left(\hat{p}_{i}\right)$. The bias of $\tilde{p}_{i}$ is seen to be of order $O\left(\mathrm{~m}^{-2}\right)$. Furthermore, the variance of the biascorrected estimator $\tilde{p}_{i}$ is of order $O\left(\mathrm{~m}^{-1}\right)$, and the $O\left(\mathrm{~m}^{-1}\right)$ term coincides with the asymptotic variance $1 / I_{i}$ of $\hat{p}_{i}$, see ${ }^{(17,18)}$.

The bias and asymptotic variance depend on the parameters $r, k$, and $l$. It is helpful to relate the general shape of these two quantities to the effort spent on inspection. Specifically, define effort as the average number of items inspected during one cycle, $\mathcal{E}_{i}:=\mathbf{E}\left(N_{i}\right)$, for $i=0, \ldots, 3$, where the $N_{i}$ are given in section 2 . The $\mathcal{E}_{i}$ s correspond to the expected value of the 

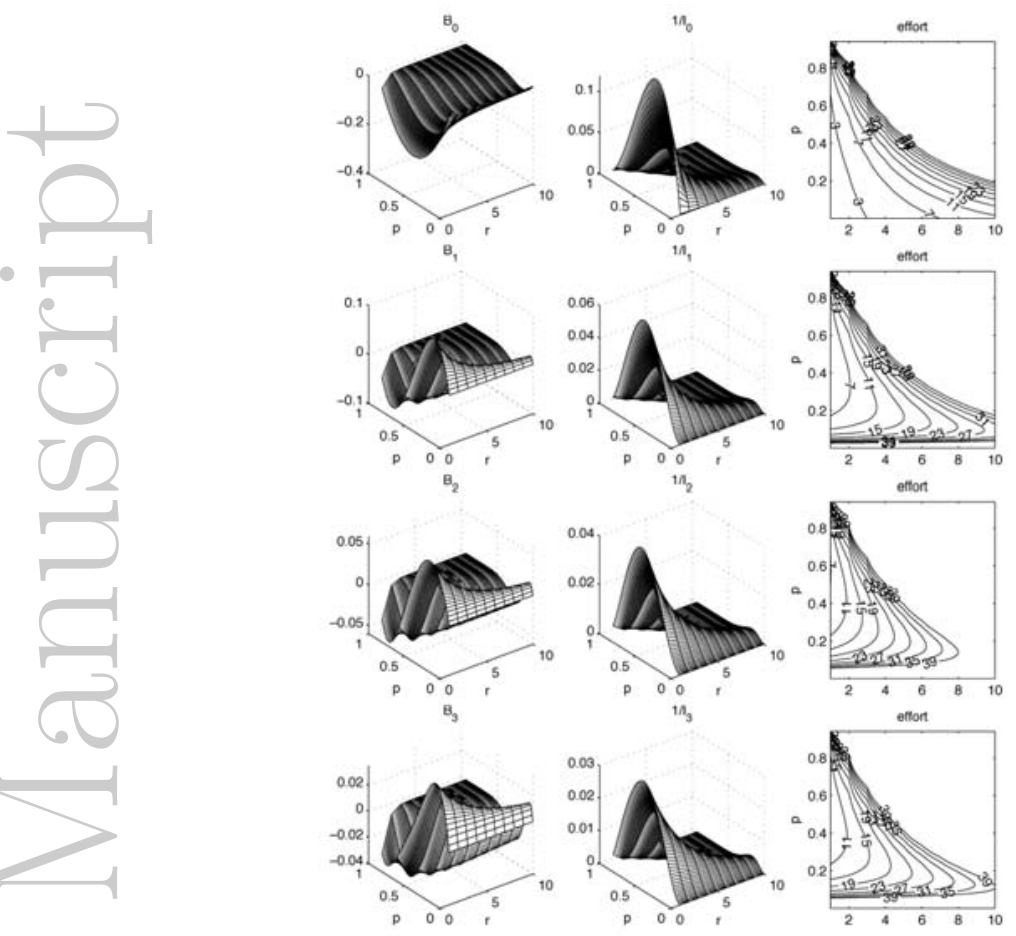

Fig. 1: Bias, variance and effort (from left to right column) for estimation at the end of a census cycle, and a full CSP-1, CSP-2 and CSP-3 cycle (from top to bottom). 
denominator in the expression of each of the $\hat{p}_{i}$. For $m=1$, we obtain,

$$
\begin{aligned}
& \mathcal{E}_{0}=\frac{1}{p q^{r}}-\frac{1}{p}, \quad \mathcal{E}_{1}=\frac{1}{p q^{r}}, \quad \mathcal{E}_{2}=\frac{1}{p q^{r}}+\frac{1}{p\left(1-q^{k}\right)}, \\
& \mathcal{E}_{3}=\frac{1}{p q^{r}}+\frac{l p+q^{l}}{p\left(1-q^{l+k}\right)} .
\end{aligned}
$$

Figure 1 plots $\mathcal{B}_{i}(p)$, the asymptotic variance expressions $I_{i}^{-1}$, and effort $\mathcal{E}_{i}$ (left to right column) for the $\hat{p}_{i}$, for the three continuous sampling plans (top to bottom). In each case, the clearance number $r$ varies between 1 and 10, and we take $m=1$. In CSP-2 and CSP-3, we take $k=10$, and $l=4$ for CSP-3, but the general shape of the bias and variance remains similar with other values of $k$ and $l$. Note that the value $l=4$ is currently implemented in some Australian quarantine inspection services. Typically, $k$ is taken equal to $r$. Estimator $\hat{p}_{0}$ suffers from a systematic negative bias. Estimation at the end of a CSP-1 cycle cancels this effect, and the bias is further reduced with CSP-2 and CSP-3. A similar pattern occurs with the variance of $\hat{p}_{i}$, it is the highest for census mode, and the smallest in CSP-3. A look at the effort spent on inspection explains this pattern: the more items are inspected, the more information we have, and the smaller the bias and the variance of $\hat{p}_{i}$.

\subsection{Numerical study}

We compare the bias and standard deviation of estimators $\hat{p}_{i}$ and their biascorrected versions $\tilde{p}_{i}$. Typically the non-compliance rate is very small in these low-risk pathways, and we focus on values of $p$ smaller than 0.05 . The simulation exercise was carried out as follows. Set $r=k=50, l=4$, and take $m=1$, corresponding to estimation at the end of the first cycle. For each CSP- $i$ cycle, we generate a sequence of independent Bernoulli random variables, with parameter $p$. At the end of each CSP- $i$ cycle, the sequence length and the number of failures is recorded, and $\hat{p}_{i}$ and $\tilde{p}_{i}$ are computed. The procedure is repeated $10^{4}$ times. The mean and standard deviation of the estimators are then empirically computed from the $10^{4}$ estimates. The results are displayed in Table I. As expected, all estimators $\hat{p}_{i}$ suffer from a bias. The relative error shows that the effect is particularly visible when the non-compliance rate is less than 0.01, a situation which arises often in quarantine inspections. On the other hand, the bias-corrected estimators reduce the bias, with only a slight increase of the variance. In some cases, 
Table I: Performance of estimators of the non-compliance rate when estimation is performed at the end of a census cycle, and at the end of a CSP-1, CSP-2 and CSP-3 cycle. The mean values the maximum likelihood estimates $\hat{p}_{i}$ and their bias-corrected versions $\tilde{p}_{i}$ are evaluated using $10^{4}$ Monte-Carlo simulations. The estimated standard deviation is given in brackets. The third line gives the relative error $\left(\hat{p}_{i}-p\right) / p$. The last line corresponds to the theoretical standard deviation of the estimator if estimation was performed when no CSP is implemented, from a sample of size $\mathcal{E}_{i}, s_{i}=\left(p(1-p) \mathcal{E}_{i}^{-1}\right)^{1 / 2}$.

\begin{tabular}{|c|c|c|c|c|}
\hline $\bar{p}$ & 0.001 & 0.005 & 0.01 & 0.05 \\
\hline \multicolumn{5}{|l|}{ Census } \\
\hline $\mathcal{E}_{0}$ & 51 & 57 & 65 & 240 \\
\hline$\hat{p}_{0}$ & $0.0007(0.0032)$ & $0.0035(0.0069)$ & $0.0071(0.0098)$ & $0.0415(0.0188)$ \\
\hline$\left(\hat{p}_{0}-p\right) / p$ & -0.3 & -0.3 & -0.29 & -0.17 \\
\hline$\tilde{p}_{0}$ & $0.0010 \quad(0.0044)$ & $0.0048(0.0093)$ & $0.0095(0.0128)$ & $0.0480(0.0198)$ \\
\hline$s_{0}$ & 0.0044 & 0.0093 & 0.0123 & 0.0141 \\
\hline \multicolumn{5}{|l|}{$\overline{\mathrm{CSP}}-1$} \\
\hline $\mathcal{E}_{1}$ & 1051 & 257 & 165 & 260 \\
\hline$\hat{p}_{1}$ & $0.0027(0.0034)$ & $0.0077(0.0057)$ & $0.0122(0.0071)$ & $0.0438(0.0153)$ \\
\hline$\left(\hat{p}_{1}-p\right) / p$ & 1.7 & 0.54 & 0.22 & -0.12 \\
\hline$\tilde{p}_{1}$ & $0.0013(0.0029)$ & $0.0055(0.0063)$ & $0.0105(0.0088)$ & $0.0483(0.0171)$ \\
\hline$s_{1}$ & 0.0010 & 0.0044 & 0.0077 & 0.00135 \\
\hline \multicolumn{5}{|l|}{ CSP-2 } \\
\hline $\mathcal{E}_{2}$ & 21,545 & 1159 & 418 & 282 \\
\hline$\hat{p}_{2}$ & $0.0014(0.0015)$ & $0.0074(0.0051)$ & $0.0133(0.0069)$ & $0.0460(0.0136)$ \\
\hline$\left(\hat{p}_{2}-p\right) / p$ & 0.4 & 0.48 & 0.33 & -0.08 \\
\hline$\tilde{p}_{2}$ & $0.0012(0.0011)$ & $0.0056(0.0048)$ & $0.0108(0.0075)$ & $0.0493(0.0154)$ \\
\hline$s_{2}$ & 0.0002 & 0.0021 & 0.0049 & 0.0130 \\
\hline \multicolumn{5}{|l|}{$\overline{\mathrm{CSP}}-3$} \\
\hline $\mathcal{E}_{3}$ & 20,065 & 1100 & 404 & 282 \\
\hline$\hat{p}_{3}$ & $0.0014(0.0016)$ & $0.0074(0.0049)$ & $0.0132(0.0068)$ & $0.0461(0.0135)$ \\
\hline$\left(\hat{p}_{3}-p\right) / p$ & 0.4 & 0.48 & 0.32 & -0.08 \\
\hline$\tilde{p}_{3}$ & $0.0012(0.0013)$ & $0.0056(0.0046)$ & $0.0108(0.0074)$ & $0.0495(0.0153)$ \\
\hline$s_{3}$ & 0.0002 & 0.0021 & 0.0050 & 0.0130 \\
\hline
\end{tabular}


corresponding to $p$ less than 0.01 , the variance is even smaller than for the original estimators.

We further compare the variability of these estimators with the standard deviation of the maximum likelihood estimator if no sampling plan was implemented, corresponding to a succession of Bernoulli trials with a fixed sample size. Taking the number of inspections to be equal to the effort $\mathcal{E}_{i}$, the standard deviation of the maximum likelihood estimator of $p$ associated with $\mathcal{E}_{i}$ Bernoulli trials is $s_{i}:=\left(p(1-p) \mathcal{E}_{i}^{-1}\right)^{1 / 2}$. Comparing the standard deviation of $\hat{p}_{i}$ with $s_{i}$ shows that in most cases (except for estimation at the end of a census cycle), estimators $\hat{p}_{i}$ have a higher variance than if no sampling plan was implemented.This indicates that the maximum likelihood estimator applied to data collected from CSPs suffer from a systematic bias and a higher variance relative to simple random sampling. The bias can be approximately corrected, and the higher variance is a reasonable price to pay for an adaptive sampling plan. The inspectorate needs to take these effects into account when estimating the non-compliance rate, or when constructing confidence intervals for $\hat{p}_{i}$.

The numerical results presented here correspond to $m=1$. Perhaps surprisingly, the results show that bias corrections developed from asymptotic likelihood theory provide useful corrections for the original estimators even for small sample size. For example, even when the sample is reduced to one observation (meaning one cycle, hence containing a lot of information about the non-compliance rate), the first-order term in the bias expression dominates higher order terms. This suggests that the magnitude of the expressions corresponding to higher order terms in the bias expansion are very small compared to the $m^{-1}$ term, as a function of $p$. We repeated these simulations for other values of $m$. For $m=2$, the variance of the estimators is indeed approximately divided by a factor 2 . The bias of the $\hat{p}_{1}$ is also much reduced compared to estimation with $m=1$, and the difference between the standard deviations of $\hat{p}_{i}$ and $\tilde{p}_{i}$ is of order $10^{-4}$.

\subsection{Robustness of the bias-corrected estimator}

We assess the robustness of the bias correction, when departing from the assumption of a constant non-compliance rate and independent observations. Specifically, the probability $p_{j}$ that the $j$-th unit is non-compliant is assumed to be equal to $p_{j}=p+\epsilon_{j}$, where $\epsilon_{j}$ is a small random perturbation with zero mean. In the numerical study to follow, to allow correlation between 
Table II: Robustness study. Reported here are the mean values of the $\hat{p}_{i} \mathrm{~s}$ and $\tilde{p}_{i} \mathrm{~s}$, and their standard deviation in brackets. The non-compliance rate is assumed non-constant, see section 4.4 for details.

\begin{tabular}{lllll}
\hline$p$ & 0.001 & 0.005 & 0.01 & 0.05 \\
\hline Census & & & & \\
$\hat{p}_{0}$ & $0.0007(0.0032)$ & $0.0036(0.0070)$ & $0.0071(0.0098)$ & $0.0412(0.0191)$ \\
$\tilde{p}_{0}$ & $0.0010(0.0044)$ & $0.0049(0.0095)$ & $0.0095(0.0128)$ & $0.0476(0.0202)$ \\
\hline$\overline{\text { CSP-1 }}$ & & & & \\
$\hat{p}_{1}$ & $0.0027(0.0034)$ & $0.0077(0.0056)$ & $0.0120(0.007)$ & $0.0436(0.0156)$ \\
$\tilde{p}_{1}$ & $0.0013(0.0030)$ & $0.0055(0.0063)$ & $0.0102(0.0086)$ & $0.0480(0.0174)$ \\
\hline$\overline{\text { CSP-2 }}$ & & & & \\
$\hat{p}_{2}$ & $0.0014(0.0014)$ & $0.0073(0.0049)$ & $0.0132(0.0068)$ & $0.0459(0.0134)$ \\
$\tilde{p}_{2}$ & $0.0012(0.0011)$ & $0.0055(0.0045)$ & $0.0107(0.0074)$ & $0.0492(0.0153)$ \\
\hline$\overline{\text { CSP-3 }}$ & & & & \\
$\hat{p}_{3}$ & $0.0014(0.0015)$ & $0.0074(0.0050)$ & $0.0131(0.0068)$ & $0.0460(0.0136)$ \\
$\tilde{p}_{3}$ & $0.0012(0.0012)$ & $0.0056(0.0047)$ & $0.0107(0.0074)$ & $0.0493(0.0154)$ \\
\hline
\end{tabular}

successive values of $p+\epsilon_{j}$, we assume that $\left\{\epsilon_{j}\right\}$ follows an autoregressive model of order $1, \epsilon_{j}=a \epsilon_{j-1}+e_{j}$, where the $e_{j}$ are i.i.d. normally distributed random variables with zero mean and variance $\sigma^{2}$. The two parameters $a$ and $\sigma^{2}$ are chosen such that the amplitude of the noise $\epsilon_{j}$ stays within $10 \%$ of the value of $p$ (namely, $\left(a, \sigma^{2}\right)=\left(10^{-6}, 5 \cdot 10^{-9}\right)$ for $p=0.001,=\left(10^{-6}, 5 \cdot 10^{-8}\right)$ for $p=0.005,=\left(10^{-5}, 10^{-7}\right)$ for $p=0.01,=\left(10^{-5}, 5 \cdot 10^{-6}\right)$ for $p=0.05$ and $=\left(10^{-4}, 10^{-5}\right)$ for $\left.p=0.1\right)$. Estimation of $\hat{p}_{i}$ and $\tilde{p}_{i}$ of $p$ at the end of the first CSP- $i$ cycle are performed the same way as in section 4.3, for the same range of values of $p$, and the same values of the CSP parameters. The only difference being that conditionally on $p_{j}=p+\epsilon_{j}$, observations are drawn according to $\mathbf{P}\left(Z_{j}=1\right)=p_{j}$. The mean and standard deviation of $\hat{p}_{i}$ and $\tilde{p}_{i}$ are estimated over $10^{4}$ Monte-Carlo simulations. The good robustness properties of the bias-corrected estimator can be seen from Table II: the bias reduction performs well with a non-constant non-compliance rate and correlated observations. A comparison with Table I indicates a similar order of bias reduction as in the i.i.d. case with constant rate. Similar performance results not presented here are obtained when the amplitude of the noise fluctuates by an amount up to $20 \%$ to $30 \%$ of the value of $p$. 


\section{Interval estimation}

In this section we construct different confidence intervals for $p$, and compare their performance in a numerical study. Consider first estimation at the end of the $m$-th CSP-1 cycle. We showed in section 4.2 that the maximum likelihood estimator $\hat{p}_{1}$ and the first-order bias corrected estimator $\tilde{p}_{1}$ both have a first-order variance of $p^{2} q^{r+1} / m$. To construct a confidence interval for $p$ with nominal coverage $(1-\alpha)$, it is common practice to rely on the asymptotic normal distribution, and take

$$
\begin{aligned}
& \hat{p}_{1} \pm z_{\alpha} m^{-1 / 2} \hat{p}_{1}\left(1-\hat{p}_{1}\right)^{(r+1) / 2}, \quad \text { or } \\
& \tilde{p}_{1} \pm z_{\alpha} m^{-1 / 2} \hat{p}_{1}\left(1-\hat{p}_{1}\right)^{(r+1) / 2},
\end{aligned}
$$

where $z_{\alpha}$ is the $100(1-\alpha / 2)$ th percentile of the standard normal distribution. The two intervals at (6) differ from the location of their centre: the left interval is centered around $\hat{p}_{1}$, while the right interval is centered around the bias corrected estimator $\tilde{p}_{1}$.

Alternatively, taking first the logit transform $\eta:=g(p):=\log (p /(1-p))$, and constructing an interval from the statistics of an estimator of $\eta$ generally improves the coverage by correcting for the asymmetry of the distribution of the maximum likelihood estimate around each end of the interval $(0,1)$.

The maximum likelihood estimator of $\eta$ at the end of a CSP- $i$ cycle is $\hat{\eta}_{i}:=g\left(\hat{p}_{i}\right)$, where $\hat{p}_{i}$ is given in (41). Note that $\hat{\eta}_{i}$ is a biased estimator of $\eta$. To evaluate the first order contribution of the bias, we proceed as before. Let $\ell_{i}^{*}(\eta)$ be the log-likelihood expressed as a function of $\eta$, so that $\ell_{i}^{*}(\eta)=$ $\ell_{i}\left(g^{-1}(\eta)\right)=\ell_{i}(p)$, where the $\ell_{i}(p)$ were introduced in section 4.1. Denoting $I_{i}^{*}:=-\mathbf{E}\left(\partial^{2} \ell_{i}^{*}(\eta) / \partial \eta^{2}\right), \mathcal{J}_{i}^{*}:=-\partial I_{i}^{*} / \partial \eta$, and $K_{i}^{*}:=\mathbf{E}\left(\partial^{3} \ell_{i}^{*}(\eta) / \partial \eta^{3}\right)$, we show in $\S 2$ on the Appendix that

$$
I_{i}^{*}=\frac{I_{i}}{\left|g^{\prime}(p)\right|^{2}}, \quad K_{i}^{*}=\frac{K_{i}}{\left|g^{\prime}(p)\right|^{3}}+3 \frac{g^{\prime \prime}(p)}{\left|g^{\prime}(p)\right|^{4}} I_{i},
$$

where the $I_{i}$ and $K_{i}$ are defined in section 4.2, so that the first-order bias-term for $\hat{\eta}_{i}$ is $\mathcal{B}_{i}^{*}:=I_{i}^{*-2}\left(\mathcal{J}_{i}^{*}-K_{i}^{*} / 2\right)$. Relation (6) hold true for any invertible function $g$, sufficiently smooth. When $g$ is taken to be the logic transform, 
we obtain for CSP-1 that $I_{1}^{*}=m / q^{r-1}, \mathcal{J}_{1}^{*}=(1-r) p m /(1-p)^{r-1}$, and

$$
\begin{aligned}
K_{1}^{*}=-2 m r p^{3} & +\frac{2 m\left(1-q^{r}\right)}{q^{r-3}} \\
& -\frac{2 m p^{2}}{q^{r}}\left(q+(r-1) q^{r+1}-r q^{r}\right) \\
& +2(1-2 p) q m+3(2 p-1) \frac{m}{q^{r-1}},
\end{aligned}
$$

so that after simplifications $\mathcal{B}_{1}^{*}(p)=(1-2 p r) / 2 q^{1-r}$. The expression of the bias terms $\mathcal{B}_{i}^{*}$ for $i=0, \ldots, 3$ corresponding to CSP-2 and CSP-3 are given in $\S 2$ of the Appendix.

For estimation at the end of a CSP-1 cycle, the bias corrected version of the maximum likelihood estimator $\hat{\eta}_{1}=g\left(\hat{p}_{1}\right)$ of $\eta$ is given by

$$
\tilde{\eta}_{1}=\hat{\eta}_{1}-m^{-1} \mathcal{B}_{1}^{*}\left(\hat{p}_{1}\right) .
$$

The first-order term in the asymptotic variance of both $\hat{\eta}_{1}$ and $\tilde{\eta}_{1}$ is $\left(I_{1}^{*}\right)^{-1}=$ $q^{r-1} / m$. Other intervals with approximate coverage $1-\alpha$ are then given by the inverse logit transform of the endpoints of

$$
\begin{aligned}
& \hat{\eta}_{1} \pm z_{\alpha} m^{-1 / 2}\left(1-\hat{p}_{1}\right)^{(r-1) / 2}, \\
& \tilde{\eta}_{1} \pm z_{\alpha} m^{-1 / 2}\left(1-\hat{p}_{1}\right)^{(r-1) / 2} .
\end{aligned}
$$

Terms $\left(I_{i}^{*}\right)^{-1}$ for $i=0, \ldots, 3$ corresponding to CSP-2 and CSP-3 are given in $\S 2$ of the Appendix.

To compare the coverage probability of the four confidence intervals defined at (6) and (9), suppose that observations collected are $0000000000001 \| 00000$ for the first cycle, and $1 \| 00000$ for the second cycle, where the double vertical bar indicates moving from sampling to census mode. The clearance number is $r=5$ for this pathway. The maximum likelihood estimate of the non-compliance rate is $\hat{p}_{1}=2 / 23 \approx 0.087$. Its bias-corrected version is $\tilde{p}_{1}=\hat{p}_{1}-\mathcal{B}_{1}\left(\hat{p}_{1}\right) m^{-1}=0.087-0.0132=0.074$, where $\mathcal{B}_{1}(p)=p q^{r}(q-p r)$. The naive estimate overestimates the non-compliance rate for this pathway. The logit transforms of $\tilde{p}_{1}$ yields

$$
\hat{\eta}_{1}=\log \left(\frac{\hat{p}_{1}}{1-\hat{p}_{1}}\right)=-2.351,
$$




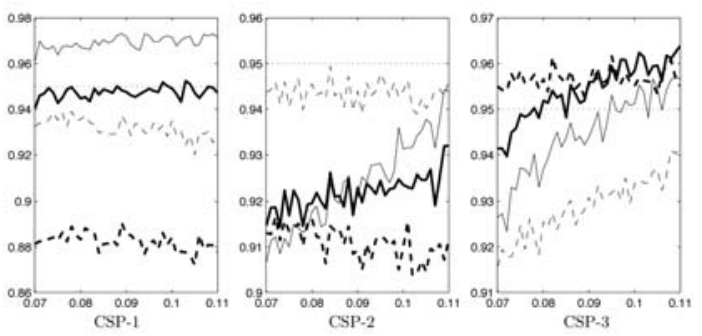

Fig. 2: Coverage probability (vertical axis) of confidence intervals (6) and (9), for $p$ varying between 0.07 and 0.11 (horizontal axis). The nominal coverage is 0.95 , represented by the dotted horizontal line. Intervals are the standard interval (dashed line) and the logit interval (plain line). Each interval is either centered around the maximum likelihood estimate (thin line) or around the bias corrected estimate (bold line).

and

$$
\tilde{\eta}_{1}=\hat{\eta}_{1}-\mathcal{B}_{1}^{*}\left(\hat{p}_{1}\right) m^{-1}=-2.374
$$

where $\mathcal{B}_{1}^{*}(p)$ is found in $\S 2$ of the Appendix. The first order term in the expansion of the variance of $\hat{\eta}_{1}$ is also given in $\S 2$ of the Appendix, so that $95 \%$ confidence intervals defined at (6) and (9) are

$$
\begin{aligned}
\hat{p}_{1} \pm z_{\alpha} I_{1}^{-1 / 2}\left(\hat{p}_{1}\right) & =\left[\begin{array}{ll}
0, & 0.179
\end{array}\right], \\
\tilde{p}_{1} \pm z_{\alpha} I_{1}^{-1 / 2}\left(\hat{p}_{1}\right) & =\left[\begin{array}{ll}
0, & 0.165
\end{array}\right], \\
g^{-1}\left(\hat{\eta}_{1} \pm z_{\alpha} I_{1}^{*-1 / 2}\left(\hat{p}_{1}\right)\right) & =\left[\begin{array}{ll}
0.029, & 0.232
\end{array}\right] \\
g^{-1}\left(\tilde{\eta}_{1} \pm z_{\alpha} I_{1}^{*-1 / 2}\left(\hat{p}_{1}\right)\right) & =\left[\begin{array}{ll}
0.028, & 0.228
\end{array}\right] .
\end{aligned}
$$

where the function $g^{-1}(x)=1 /(1+\exp (-x))$ is the inverse logit transform. Since our approach is parametric, decision on which interval to use can be made by comparing the coverage properties of the four intervals in a neighbourhood of the point estimate, for example here over the interval $[0.08,0.11]$. The left panel in Figure 2 presents the results of the numerical study, where the coverage probability is estimated from $10^{4}$ Monte-Carlo simulations. In this situation, it is reasonable to keep the logit interval. The situation would be rather different if, for the same range of non-compliance rates, CSP-2 or -3 was implemented, obtained with $r=k=5$ and $l=4$. In the particular case of CSP-3, the right panel of Figure 2 shows that the two 

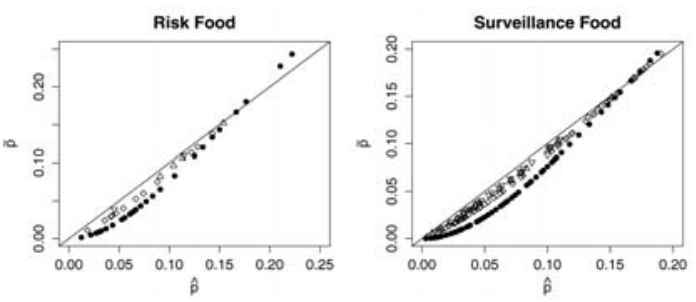

Fig. 3: Scatterplot of $\tilde{p}$ versus $\hat{p}$ for risk food and surveillance food, for estimation at the end of the $m$-th cycle, with $m=1(\circ), 2(\diamond), 3(\triangle)$ and 4 $(\nabla)$.

intervals centered around the bias corrected estimate have better coverage here.

\section{Border biosecurity inspections}

We focus on a scheme run by the department called the Imported Food Inspection Scheme (IFIS). Imported foods are grouped into two categories, called risk food and surveillance food. Classification is based on economical and ecological consequences of a potential contamination, with risk food products having substantially more severe consequences than surveillance food products. For risk food, the scheme runs as follows

1. Census mode. Inspect all units as they arrive until 5 consecutive units pass inspection, then switch to sampling mode 1.

2. Sampling mode 1. While the number of items to pass inspection is less than $\kappa$, and no items fail inspection, inspect $25 \%$ of incoming units. If an item fails inspection, switch back to census mode. Otherwise, when $\kappa$ items have passed inspection, switch to sampling mode 2.

3. Sampling mode 2 . Inspect $5 \%$ of incoming units until one failure occurs and go back to census.

The department uses $\kappa=20$ for IFIS. Units grouped into surveillance food follow a slightly modified version of the previous procedure,

1. Sampling mode. Inspect $5 \%$ of incoming units until a non-compliant unit occurs. Switch to step 2. 
2. Census mode. Inspect all units until 5 consecutive units pass inspection.

We shall refer to each run of the risk food or surveillance food algorithm as a cycle. In addition to the overall inspection result, IFIS data contains considerable contextual information, such as the country of origin, the identity of the producer, or the method of inspection.

Under the independence assumption of observations, we show in $\S 3$ of the Appendix that the first-order bias term for risk food is the same as for the CSP-1 scheme, namely $\mathcal{B}_{1}(p)$. Moreover, this holds true as well for the surveillance food sampling scheme: when estimation is performed at the end of a full cycle, the order of the two modes (census followed by sampling, or sampling followed by census) does not matter. As such, the first-order bias for surveillance food is $\mathcal{B}_{1}(p)$ as well.

For the six and a half year span from January 1st, 2008 until May 1st, 2014 , the data consists of 557,607 observations, split into 225,186 observations for risk food (47,149 distinct pathways), and 332,419 observations for surveillance food (40,932 pathways). A sampling plan is applied to each pathway. For each pathway, we extract information about a full cycle (sampling and census). These can be easily identified since the beginning of a sampling mode/end of a census mode corresponds to the occurrence of the sequence FPPPPP (the clearance number is $r=5$ here). The total number of full cycles differ from one pathway to another, and we perform estimation at the end of the last full cycle.

Scatter plots of $\tilde{p}$ versus $\hat{p}$ are presented in Figure 3. Pathways with noncompliance rate lower than 0.15 usually overestimate the true rate, while the opposite occurs for the few higher-risk pathways. Further, it can be seen from Table III that the proportion of pathways with estimated noncompliance rate no higher than a given threshold is greatly affected by the bias-correction.

For each pathway, we use the Average Output Quality (AOQ) as an indicator of the health of the pathway. If $F$ denotes the fraction of inspected items per cycle, then the AOQ is defined as $p(1-F)$, see Dodge (1943). It is customary in CSP designs to choose or update the parameters of the algorithm so that the AOQ stays below a specified target $\mathrm{AOQ}^{*}$. We focus on the surveillance food algorithm first: two parameters need to be chosen: the clearance number $r$, and the sampling frequency $f$. Parameters $r=5$ and $f=0.05$ are set for the department by federal legislation. The department may seek to update these two parameters, to ensure that each pathway stays 
Table III: Proportion of pathways with estimated non-compliance rate less than 0.02, 0.05 and 0.1, based on the maximum likelihood estimator $\hat{p}$, and on the bias-corrected estimator $\tilde{p}$.

\begin{tabular}{lccc}
\hline & $\leq 0.02$ & $\leq 0.05$ & $\leq 0.1$ \\
\hline Risk food & & & \\
$\hat{p}$ & $4.5 \%$ & $21.4 \%$ & $67.8 \%$ \\
$\tilde{p}$ & $17.8 \%$ & $51.8 \%$ & $75.9 \%$ \\
\hline Surveillance food & & & \\
$\hat{p}$ & $11.7 \%$ & $41.6 \%$ & $79.7 \%$ \\
$\tilde{p}$ & $37.1 \%$ & $66.6 \%$ & $83.8 \%$ \\
\hline
\end{tabular}
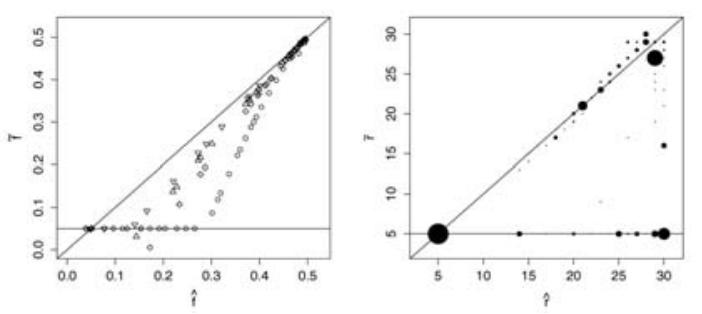

Fig. 4: Scatterplot of $\tilde{f}$ (resp. $\tilde{r}$ ) versus $\hat{f}$ (resp. $\hat{r}$ ) for surveillance food, for estimation at the end of the $m$-th cycle, with $m=1(\circ), 2(\diamond), 3(\triangle)$ and 4 $(\nabla)$. The size of the dots on the right panel are proportional to the number of observations. The size of the dot at $\hat{r}=\tilde{r}=5$ is scaled down to keep the figure readable. 
below AOQ* . For CSP-1, the average fraction $F$ of inspected items per cycle is

$$
F=\frac{\mathbf{E}\left(N_{0}+S\right)}{\mathbf{E}\left(N_{0}+S / f\right)}=\frac{f}{f+q^{r}(1-f)},
$$

since in the notation of section 2.2, the average number of inspected items is $\mathbf{E}\left(N_{0}+S\right)$, while the average number of items that have passed for inspection is $\mathbf{E}\left(N_{0}+S / f\right)$, as only a fraction $f$ of items are inspected in sampling mode. We obtain

$$
A O Q(r, f, p):=p(1-F)=\frac{p q^{r}(1-f)}{f+q^{r}(1-f)},
$$

where $0<f<1$ and $r \geq 1$. For each fixed $p, A O Q(r, f, p)$ is a strictly decreasing function of both $r$ and $f$. We consider updating either $r$ of $f$ to meet the target $\mathrm{AOQ}^{*}=0.05$, keeping the other parameter to its current value. In particular, we evaluate the difference in the needed change of policy, depending on whether decisions are made based on $\hat{p}$ or $\tilde{p}$. We proceed as follows. If $A O Q^{*}<A O Q(r=5,0, \hat{p})$, (respectively $A O Q^{*}<A O Q(1, f=$ $0.05, \hat{p})$ ), find the smallest $f$ to meet the target $A O Q^{*}$ (resp. find the smallest $r$ ), otherwise, keep $f=0.05$ (resp. keep $r=5$ ). The procedure is then repeated with $\tilde{p}$ instead of $\hat{p}$. Denote updated values by $\hat{f}$ and $\hat{r}$ (resp. $\tilde{f}$ and $\tilde{r})$.

Scatterplots of $\tilde{f}$ (resp. $\tilde{r}$ ) versus $\hat{f}$ (resp. $\hat{r}$ ) for each pathway in the surveillance food category are presented in Figure 4. Any departure from the diagonal line indicates a different change of policy depending on whether updates are based on $\hat{p}$ or $\tilde{p}$. In $34 \%$ of the time $(\operatorname{resp} .37 \%), \tilde{f}=\hat{f}=0.05$ (resp. $\tilde{r}=\hat{r}=5$ ), while $38 \%$ (resp. 50\%) of the pathways return $\tilde{f}=\hat{f}$ (resp. $\tilde{r}=\hat{r}$ ). In several occasions, the bias-corrected estimate remains at the current value parameters, while inference based on $\hat{p}$ would suggest a change of policy. This situation occurs when the AOQ is necessary less than the target when calculated with $\tilde{p}$, but not with $\hat{p}$. In general, it can be seen that the change of policy tends to be too conservative for decisions taken with $\hat{p}$, which emphasizes the importance of the bias correction. In particular, considerable savings in terms of costs associated on inspections can be made if $\tilde{f}$ or $\tilde{r}$ are used instead of $\hat{f}$ or $\hat{r}$.

The expression of the AOQ for risk food is slightly different than for surveillance food, due to the presence of two distinct sampling rates $f_{1}=0.25$ and $f_{2}=0.05$ in sampling modes 1 and 2 . We derive its expression in $\S 4$ of 
the Appendix, where it is shown that

$$
\begin{aligned}
& \operatorname{AOQ}\left(r, \kappa, f_{1}, f_{2}, p\right) \\
& =\frac{p q^{r}\left\{\left(f_{1}-f_{2}\right) q^{\kappa}+f_{2}\left(1-f_{1}\right)\right\}}{f_{1} f_{2}+q^{r}\left\{\left(f_{1}-f_{2}\right) q^{\kappa}+f_{2}\left(1-f_{1}\right)\right\}},
\end{aligned}
$$

where $0<f_{1}, f_{2}<1$, and $r, \kappa \geq 1$. The same protocol as for surveillance food can then be applied, and similar conclusions can be made, but to keep the exposition short, the results are not presented.

\section{Acknowledgment}

The authors are grateful to Richard Gao of the Australian Federal Department of Agriculture and Water Resources for providing the example data, and to Martina Hoffmann for assistance in identifying applications of the CSP methodology. The authors acknowledge funding support from CEBRA and ARC Discovery Project DP110103159.

\section{REFERENCES}

[1] Dodge HF. A sampling inspection plan for continuous production. The Annals of Mathematical Statistics, 1943; 22:264-279.

[2] Dodge HF, Torrey MN. Additional continuous sampling inspection plans. Industrial Quality Control, 1951; 7:5-9.

[3] United State Department of Defence. Military Standard, Single and Multi-level Continuous Sampling Procedures and Tables for Inspection by Attributes, Functional Curves of the Continuous Sampling Plans. ASTM E2819 standard, USA, 1998.

[4] King DW, Lashley R. A quantifiable alternative to double data entry. Controlled clinical trials, 2000; 21: 94-102.

[5] Kern R, Thies H, Satzger G. Statistical quality control for human-based electronic services. Service-Oriented Computing. Springer Berlin Heidelberg, 2010; 243-257. 
[6] Groomer SM, Murthy US. Monitoring high volume on-line transaction processing systems using a continuous sampling approach. International Journal of Auditing, 2003; 7:3-19.

[7] Antila J, Karhu T. A comparative study of continuous sampling plans for functional board testing. Test Conference, 2007. ITC 2007. IEEE International, 2007; 1-7.

[8] Decrouez G, Robinson A. Time series models for border inspection data. Risk Analysis: An International Journal, 2013; 33:2142-2153.

[9] Kumar VS, Rajarshi MB. Continuous sampling plans for MarkovDependent production processes. Naval Research Logistics, 1987; 34:629-644.

[10] Balamurali S, Subramali K. Modified CSP-C continuous sampling plan for consumer protection. Journal of Applied Statistics, 2004; 31:481-494.

[11] Balamurali S, Jun CH. Average outgoing quality of CSP-C continuous sampling plan under short run production process. Journal of Applied Statistics, 2006; 33:139-154.

[12] Cao Y, Subramaniam V. Improving the performance of manufacturing systems with continuous sampling plans. IIE Transactions, 2013; 45:575590.

[13] Eleftheriou M, Farmakis N. Expected cost for continuous sampling plans. Communications in Statistics. Theory and Methods, 2011; 40:2969-2984.

[14] Kumar VS, Cassady CR. Assessing the economic performance of continuous sampling plans. Qual. Technol. Quantit. Manage, 2006; 3:45-54.

[15] Cox DR, Snell EJ. A general definition of residuals. Journal of the Royal Statistical Society. Series B, 1968; 30:248-275.

[16] Cordeiro GM, Klein R. Bias correction in ARMA models. Statistics and Probability Letters, 1994; 169-176.

[17] Ferrari SLP, Botter DA, Cordeiro GM, Cribari-Neto F. Second- and third-order bias reduction for one-parameter family models. Statistics and Probability Letters, 1996; 30:339-345. 
[18] Pace L, Salvan A. Principles of Statistical Inference. World Scientific, Singapore, 1997.

\section{A APPENDIX}

\section{A.1 Distribution of $\mathrm{L}_{3}$}

We first derive the joint distribution of $\left(N_{l}, N_{a}\right)$. The number of limbo and alert modes can only differ by 1 . The support of the joint distribution of $\left(N_{l}, N_{a}\right)$ is thus $\mathcal{S}=\{(i, j) \mid i \geq 1, j \geq 0, i=j+1$ or $i=j\}$, and $\mathbf{P}\left(N_{l}=\right.$ $\left.i, N_{a}=i\right)=q^{l} q^{(k+l)(i-1)}\left(1-q^{k}\right), \mathbf{P}\left(N_{l}=i, N_{a}=i-1\right)=q^{(k+l)(i-1)}\left(1-q^{l}\right)$, for $i \geq 1$. It follows that

$$
\mathbf{P}\left(N_{a}=N_{l}\right)=\sum_{i \geq 1} \mathbf{P}\left(N_{l}=i, N_{a}=i\right)=\frac{\left(1-q^{k}\right) q^{l}}{1-q^{k+l}}
$$

and

$$
\mathbf{P}\left(N_{a} \neq N_{l}\right)=1-\mathbf{P}\left(N_{a}=N_{l}\right)=\frac{1-q^{l}}{1-q^{k+l}} .
$$

Note that the event $\left\{N_{a}=N_{l}\right\}$ corresponds to the event of returning to census from alert mode, and that the event $\left\{N_{a} \neq N_{l}\right\}$ coincides with the event of returning to census from limbo mode. The marginal distribution of $N_{l}$ is geometric with parameter $q^{k+l}$,

$$
\begin{aligned}
\mathbf{P}\left(N_{l}=i\right)= & \mathbf{P}\left(N_{l}=i, N_{a}=i\right) \\
& \quad+\mathbf{P}\left(N_{l}=i, N_{a}=i-1\right) \\
= & \left(1-q^{k+l}\right) q^{(i-1)(k+l)},
\end{aligned}
$$

for $i \geq 1$. It follows that $\mathbf{E}\left(N_{l}\right)=1 /\left(1-q^{k+l}\right)$, and $\mathbf{E}\left(S^{N_{l}}\right)=\mathbf{E}\left(N_{l}\right) \mathbf{E}\left(S_{1}\right)=$ $1 /\left(p\left(1-q^{k+l}\right)\right)$. Similarly, the marginal distribution of $N_{a}$ satisfies $\mathbf{P}\left(N_{a}=\right.$ $0)=1-q^{l}$, and $\mathbf{P}\left(N_{a}=i\right)=q^{-k}\left(1-q^{k+l}\right) q^{i(k+l)}$, for $i \geq 1$. Its mean is $q^{l} /\left(1-q^{k+l}\right)$.

We now turn our attention to the distribution of $W_{a}$ and $W_{l}$. Their distribution depend on the values taken by $N_{a}$ and $N_{l}$ : the probability that $W_{a}$ is zero is exactly the probability that we return to census mode from limbo mode,

$$
\mathbf{P}\left(W_{a}=0\right)=\mathbf{P}\left(N_{a} \neq N_{l}\right)=\frac{1-q^{l}}{1-q^{k+l}} .
$$

This article is protected by copyright. All rights reserved. 
Similarly, for $1 \leq w \leq k$,

$$
\begin{aligned}
\mathbf{P}\left(W_{a}=w\right) & =\mathbf{P}\left(W_{a}=w \mid N_{a}=N_{l}\right) \mathbf{P}\left(N_{a}=N_{l}\right) \\
& =\frac{p q^{w-1}}{1-q^{k}} \frac{\left(1-q^{k}\right) q^{l}}{1-q^{k+l}}=\frac{p q^{w+l-1}}{1-q^{k+l}}
\end{aligned}
$$

The mean of $W_{a}$ is $\mathbf{E}\left(W_{a}\right)=q^{l}\left(1-q^{k}-k p q^{k}\right) /\left(p\left(1-q^{k+l}\right)\right)$. The distribution of $W_{l}$ is derived in a similar way,

$$
\mathbf{P}\left(W_{l}=0\right)=\mathbf{P}\left(N_{a}=N_{l}\right)=\frac{\left(1-q^{k}\right) q^{l}}{1-q^{k+l}},
$$

and, for $1 \leq w \leq l$

$$
\begin{aligned}
\mathbf{P}\left(W_{l}=w\right) & =\mathbf{P}\left(W_{a}=w \mid N_{a} \neq N_{l}\right) \mathbf{P}\left(N_{a} \neq N_{l}\right) \\
& =\frac{\left(\begin{array}{l}
l \\
w
\end{array}\right) p^{w}(1-p)^{l-w}}{1-q^{l}} \frac{1-q^{l}}{1-q^{k+l}} \\
& =\frac{\left(\begin{array}{l}
l \\
w
\end{array}\right) p^{w}(1-p)^{l-w}}{1-q^{k+l}}
\end{aligned}
$$

The mean of $W_{l}$ is $\mathbf{E}\left(W_{l}\right)=l p /\left(1-q^{k+l}\right)$.

\section{A.2 Variance and bias correction for the logic trans- form}

In this section we first derive expression (8), which shows how the expected value of the third derivative of the log-likelihood function is changed under a non-linear transform. Let $\theta$ be the parameter of interest of parametric family of distributions with density $f$. Denote by $\ell(\theta):=\log f(\theta)$ the log-likelihood, and let $\hat{\theta}$ be the maximum likelihood estimator of $\theta$. Then

$$
\mathbf{E}\left(\frac{\partial \ell(\theta)}{\partial \theta}\right)=\mathbf{E}\left(\frac{f^{\prime}(\theta)}{f(\theta)}\right)=0
$$

and

$$
I(\theta):=-\mathbf{E}\left(\frac{\partial^{2} \ell(\theta)}{\partial \theta^{2}}\right)=-\mathbf{E}\left(\frac{f^{\prime \prime}(\theta)}{f(\theta)}-\left(\frac{f^{\prime}(\theta)}{f(\theta)}\right)^{2}\right)
$$


Differentiating the log-likelihood a third time yields

$$
\begin{aligned}
K(\theta) & :=\mathbf{E}\left(\frac{\partial^{3} \ell(\theta)}{\partial \theta^{3}}\right) \\
& =\mathbf{E}\left(2\left(\frac{f^{\prime}(\theta)}{f(\theta)}\right)^{3}-3 \frac{f^{\prime}(\theta) f^{\prime \prime}(\theta)}{(f(\theta))^{2}}+\frac{f^{\prime \prime \prime}(\theta)}{f(\theta)}\right) .
\end{aligned}
$$

Suppose that $g$ is a strictly increasing and continuous mapping, with inverse $g^{-1}$, and put $\eta=g(\theta)$. Then $\hat{\eta}=g(\hat{\theta})$ is the maximum likelihood estimator of $\eta$, and $\ell^{*}(\eta):=\log f^{*}(\eta)=\ell\left(g^{-1}(\eta)\right)$ denotes the $\log$-likelihood of the variable $\eta$. Put

$$
I^{*}(\eta):=-\mathbf{E}\left(\frac{\partial^{2} \ell^{*}(\eta)}{\partial \eta^{2}}\right), \quad K^{*}(\eta)=\mathbf{E}\left(\frac{\partial^{3} \ell(\eta)}{\partial \eta^{3}}\right)
$$

We express $I^{*}(\eta)$ and $K^{*}(\eta)$ in terms of $I(\theta)$ and $K(\theta)$. One has

$$
\begin{aligned}
\frac{\partial \ell^{*}(\eta)}{\partial \eta} & =\frac{\partial \log f\left(g^{-1}(\eta)\right)}{\partial \eta} \\
& =\frac{f^{\prime}\left(g^{-1}(\eta)\right)}{f\left(g^{-1}(\eta)\right)} \frac{d g^{-1}(\eta)}{d \eta}=\frac{f^{\prime}(\theta)}{f(\theta)} \frac{1}{g^{\prime}(\theta)} .
\end{aligned}
$$

and

$$
\begin{aligned}
& \frac{\partial^{2} \ell^{*}(\eta)}{\partial \eta^{2}}=\frac{1}{\left|g^{\prime}(\theta)\right|^{2}} \\
& \times\left(-\left(\frac{f^{\prime}(\theta)}{f(\theta)}\right)^{2}+\frac{f^{\prime \prime}(\theta)}{f(\theta)}-\frac{g^{\prime \prime}(\theta)}{g^{\prime}(\theta)} \frac{f^{\prime}(\theta)}{f(\theta)}\right) .
\end{aligned}
$$

Taking expectations of (A.2), and comparing with the definition of $I(\theta)$ at (A.1) yields

$$
I^{*}(\eta)=-\mathbf{E}\left(\frac{\partial^{2} \ell^{*}(\eta)}{\partial \eta^{2}}\right)=\frac{I(\theta)}{\left|g^{\prime}(\theta)\right|^{2}}
$$


Differentiating expression (A.2) once more with respect to $\eta$, and making use of the chain rule, yields

$$
\begin{aligned}
& \frac{\partial^{3} \ell^{*}(\eta)}{\partial \eta^{3}}=\frac{f^{\prime}(\theta)}{f(\theta)}\left(3 \frac{\left(g^{\prime \prime}(\theta)\right)^{2}}{\left(g^{\prime}(\theta)\right)^{5}}-\frac{g^{\prime \prime \prime}(\theta)}{\left(g^{\prime}(\theta)\right)^{4}}\right) \\
& +\frac{1}{\left(g^{\prime}(\theta)\right)^{3}}\left(2\left(\frac{f^{\prime}(\theta)}{f(\theta)}\right)^{3}-3 \frac{f^{\prime}(\theta) f^{\prime \prime}(\theta)}{(f(\theta))^{2}}+\frac{f^{\prime \prime \prime}(\theta)}{f(\theta)}\right) \\
& +3 \frac{g^{\prime \prime}(\theta)}{\left(g^{\prime}(\theta)\right)^{4}}\left(\left(\frac{f^{\prime}(\theta)}{f(\theta)}\right)^{2}-\frac{f^{\prime \prime}(\theta)}{f(\theta)}\right) .
\end{aligned}
$$

Taking the expected value of (A.3) yields

$$
K^{*}(\eta)=\frac{K(\theta)}{\left|g^{\prime}(p)\right|^{3}}+3 \frac{g^{\prime \prime}(p)}{\left|g^{\prime}(p)\right|^{4}} I(\theta),
$$

as required.

Next, we summarize the expressions of the asymptotic variance of $\hat{\eta}_{i}=$ $\log \left(\hat{p}_{i} /\left(1-\hat{p}_{i}\right)\right)$, where $\hat{p}_{i}$ is the maximum likelihood estimator of $p$ at the end of a census cycle $(i=0)$, or at the end of a CSP- $i$ cycle $(i=1,2,3)$. The terms $\mathcal{B}_{i}^{*}(p)$ are also provided to construct the bias-corrected estimators $\tilde{\eta}_{i}=\hat{\eta}_{i}-\mathcal{B}_{i}^{*}\left(\hat{p}_{i}\right)$. The first order term $1 / I_{i}^{*}(p)$ in the expansion of the variance of $\tilde{\eta}_{i}$ corresponds to the asymptotic variance of $\hat{\eta}_{i}$. We have

$$
\begin{gathered}
\left(I_{0}^{*}\right)^{-1}=\frac{1}{1-q^{r}} \frac{q^{r-1}}{m}, \\
\left(I_{1}^{*}\right)^{-1}=\frac{q^{r-1}}{m}, \\
\left(I_{2}^{*}\right)^{-1}=\frac{1-q^{k}}{1-q^{k}+q^{r}} \frac{q^{r-1}}{m}, \\
\left(I_{3}^{*}\right)^{-1}=\frac{1-q^{k+l}}{1-q^{k+l}+q^{r}\left(l p+q^{l}\right)} \frac{q^{r-1}}{m},
\end{gathered}
$$

This article is protected by copyright. All rights reserved. 
and

$$
\begin{aligned}
\mathcal{B}_{0}^{*}(p) & =\frac{\left(1-2 p r-q^{r}\right)}{2\left(1-q^{r}\right)^{2}} q^{r-1} \frac{q^{r-1}}{m} \\
\mathcal{B}_{1}^{*}(p) & =\frac{(1-2 p r)}{2} q^{r-1} \\
\mathcal{B}_{2}^{*}(p) & =\frac{2 k p q^{r+k}+\left(1-q^{k}\right)\left(q^{r}+(1-2 p r)\left(1-q^{k}\right)\right)}{2 q^{1-r}\left(1-q^{k}+q^{r}\right)^{2}} \\
\mathcal{B}_{3}^{*}(p) & =\frac{2 p q^{r+k+l}\left(l^{2} p+k q\right)-l p q^{r} \mathcal{Q}+\left(1-q^{k+l}\right) \mathcal{Q}^{\prime}}{2 q^{1-r}\left(1-q^{k+l}+q^{r+l}+l p q^{r}\right)^{2}}
\end{aligned}
$$

where $\mathcal{Q}=1-2 q^{l}-q^{k+l}-2 p\left(1-q^{k+l}+k q^{k+l}\right)$ and $\mathcal{Q}^{\prime}=1-q^{k+l}+q^{r+l}-$ $2 \operatorname{pr}\left(1-q^{k+l}\right)$.

\section{A.3 Risk food scheme}

We show that for the sampling scheme introduced in $\S 6$ used for units grouped into Risk Food (RF), the expression of the first order bias term of the maximum likelihood estimator of $p$ is the same as for CSP-1.

Let $S_{1}$ be the number of observations in sampling mode 1 , and $S_{2}$ the number of inspected items during sampling mode 2 . These are random variables, and take values in $\{1,2, \ldots, \kappa\}$ and $\{0,1,2, \ldots\}$, respectively. A census cycle is described as before by the random vector $\mathbf{X}=\left(X_{1}, \ldots, X_{r}\right)$, which follows a negative multinomial distribution, see section 3.1 .

Distribution of $\left(S_{1}, S_{2}\right)$. Under the Bernoulli assumption, the joint distribution of $\left(S_{1}, S_{2}\right)$ is such that $\mathbf{P}\left(S_{1}=s_{1}, S_{2}=s_{2}\right)=0$ if $1 \leq s_{1} \leq \kappa-1$ and $s_{2} \geq 1$, or $\left(s_{1}, s_{2}\right)=(\kappa, 0)$, and $\mathbf{P}\left(S_{1}=s_{1}, S_{2}=s_{2}\right)=p q^{s_{1}+s_{2}-1}$ if $s_{1}=\kappa$ and $s_{2} \geq 1$, or $1 \leq s_{1} \leq \kappa-1$ and $s_{2}=0$. The marginal distributions of $S_{1}$ and $S_{2}$ are respectively,

$$
\mathbf{P}\left(S_{1}=s_{1}\right)= \begin{cases}p q^{s_{1}-1} & \text { if } 1 \leq s_{1} \leq \kappa-1 \\ p q^{\kappa-1}+q^{\kappa} & \text { if } s_{1}=\kappa\end{cases}
$$

and

$$
\mathbf{P}\left(S_{2}=s_{2}\right)= \begin{cases}1-q^{\kappa} & \text { if } s_{2}=0 \\ p q^{\kappa+s_{2}-1} & \text { if } s_{1} \geq 1\end{cases}
$$

from which it follows that $\mathbf{E}\left(S_{1}\right)=\left(1-q^{\kappa}\right) / p$ and $\mathbf{E}\left(S_{2}\right)=q^{\kappa} / p$. 
Maximum likelihood estimator. Consider estimation of $p$ at the end of the $m$-th $\mathrm{RF}$ cycle. Let $\mathbf{X}_{1}, \ldots, \mathbf{X}_{m}$ be a random sample of size $m$, where each $\mathbf{X}_{i}$ is distributed according to (1). Let $\ell_{R F}(p)$ be the log-likelihood of a random sample of size $m$ corresponding to the RF algorithm. Then,

$$
\ell_{R F}(p)=\ell_{0}(p)+\xi_{R F} \log p+\zeta_{R F} \log (1-p),
$$

where $\ell_{0}$ denotes the log-likelihood given by (21), and $\xi_{R F}=m, \zeta_{R F}=$ $\sum_{i=1}^{m}\left(S_{1, i}+S_{2, i}\right)-m$, where $\left(S_{1, i}, S_{2, i}\right)$ are independent copies of $\left(S_{1}, S_{2}\right)$, corresponding to observations from the $i$-th RF cycle. The maximum likelihood estimator of the approach rate at the end of the $m$-th cycle is

$$
\hat{p}=\frac{\sum_{j=1}^{r} X_{(j)}+m}{m r+\sum_{j=1}^{r} j X_{(j)}+\sum_{i=1}^{m}\left(S_{1, i}+S_{2, i}\right)},
$$

which corresponds to the ratio of non-compliant items to the total number of inspected items.

First-order bias. Recall from section 4.2 that the first order bias $\mathcal{B}(p)$ of $\hat{p}$ is given by $\mathcal{B}(p)=I^{-2}(\mathcal{J}-K / 2)$, where

$$
I=-\mathbf{E}\left(\frac{\partial^{2} \ell_{R F}(p)}{\partial p^{2}}\right), \quad K=\mathbf{E}\left(\frac{\partial^{3} \ell_{R F}(p)}{\partial p^{3}}\right),
$$

and $\mathcal{J}=-\partial I / \partial p$. The second and third order derivative of $\ell_{R F}(p)$ with respect to $p$ are given by

$$
\begin{aligned}
& \frac{\partial^{2} \ell_{R F}(p)}{\partial^{2} p}=\frac{\partial^{2} \ell_{0}(p)}{\partial^{2} p}-\frac{\xi_{R F}}{p^{2}}-\frac{\zeta_{R F}}{(1-p)^{2}}, \\
& \frac{\partial^{3} \ell_{R F}(p)}{\partial^{3} p}=\frac{\partial^{3} \ell_{0}(p)}{\partial^{3} p}+\frac{2 \xi_{R F}}{p^{3}}-\frac{2 \zeta_{R F}}{(1-p)^{3}} .
\end{aligned}
$$

Taking expectations of these expressions yields $I=m / p^{2} q^{r+1}$, and

$$
\begin{aligned}
K=-\frac{2 m r}{q^{3}}+\frac{2 m\left(1-q^{r}\right)}{p^{3} q^{r}}+\frac{2(1-2 p) m}{p^{3} q^{2}} \\
-\frac{2 m}{p q^{r+3}}\left(q+(r-1) q^{r+1}-r q^{r}\right),
\end{aligned}
$$

which corresponds to $I_{1}$ and $K_{1}$, derived for estimation at the end of a CSP-1 cycle, see $\S 4.2$. The first-order bias expression for estimation of $p$ at the end of the $m$-th RF cycle is thus the same as for estimation at the end of CSP-1 cycle, namely $\mathcal{B}_{1}(p)=p q^{r}(q-p r)$. 


\section{A.4 AOQ for risk food}

Making use of notation of $\S 3$ of the Appendix, the average fraction $F$ of inspect units in one risk food cycle is

$$
F=\frac{\mathbf{E}\left(N_{0}+S_{1}+S_{2}\right)}{\mathbf{E}\left(N_{0}+S_{1} / f_{1}+S_{2} / f_{2}\right)},
$$

where $\mathbf{E}\left(N_{0}\right)=\mathcal{E}_{0}=\left(1-q^{r}\right) / p q^{r}, \mathbf{E}\left(S_{1}\right)=\left(1-q^{\kappa}\right) / p$ and $\mathbf{E}\left(S_{2}\right)=q^{\kappa} / p$, see $\S 4.2$ and $\S 3$ of the Appendix. After simplifications, we get

$$
F=\frac{f_{1} f_{2}}{f_{1} f_{2}\left(1-q^{r}\right)+f_{2} q^{r}\left(1-q^{\kappa}\right)+f_{1} q^{r} q^{\kappa}} .
$$

The AOQ is then defined as $A O Q\left(r, \kappa, f_{1}, f_{2}, p\right):=p(1-F)$. We obtain

$$
\begin{aligned}
& \operatorname{AOQ}\left(r, \kappa, f_{1}, f_{2}, p\right) \\
& =\frac{p q^{r}\left\{\left(f_{1}-f_{2}\right) q^{\kappa}+f_{2}\left(1-f_{1}\right)\right\}}{f_{1} f_{2}+q^{r}\left\{\left(f_{1}-f_{2}\right) q^{\kappa}+f_{2}\left(1-f_{1}\right)\right\}},
\end{aligned}
$$

where $0<f_{1}, f_{2}<1$, and $r, \kappa \geq 1$. 


\section{University Library}

\section{- M M I E R R A A gateway to Melbourne's research publications}

Minerva Access is the Institutional Repository of The University of Melbourne

Author/s:

Decrouez, G;Robinson, A

Title:

Bias-Corrected Estimation in Continuous Sampling Plans

Date:

2018-01-01

Citation:

Decrouez, G. \& Robinson, A. (2018). Bias-Corrected Estimation in Continuous Sampling Plans. RISK ANALYSIS, 38 (1), pp.177-193. https://doi.org/10.1111/risa.12811.

Persistent Link:

http://hdl.handle.net/11343/292948 